doi:10.13108/2015-7-2-102

\title{
EXISTENCE OF HYPERCYCLIC SUBSPACES FOR TOEPLITZ OPERATORS
}

\author{
A.A. LISHANSKII
}

In this work we construct a class of coanalytic Toeplitz operators, which have an infinitedimensional closed subspace, where any non-zero vector is hypercyclic. Namely, if for a function $\varphi$ which is analytic in the open unit disc $\mathbb{D}$ and continuous in its closure the conditions $\varphi(\mathbb{T}) \cap \mathbb{T} \neq \emptyset$ and $\varphi(\mathbb{D}) \cap \mathbb{T} \neq \emptyset$ are satisfied, then the operator $\varphi\left(S^{*}\right)$ (where $S^{*}$ is the backward shift operator in the Hardy space) has the required property. The proof is based on an application of a theorem by Gonzalez, Leon-Saavedra and Montes-Rodriguez.

Keywords: Toeplitz operators, hypercyclic operators, essential spectrum, Hardy space.

\section{INTRODUCTION}

Let $X$ be a separable Banach space (or a Frechet space), and let $T$ be a bounded linear operator in $X$. If there exists $x \in X$ such that the set $\left\{T^{n} x, n \in \mathbb{N}_{0}\right\}$ is dense in $X$, then $T$ is said to be a hypercyclic operator and $x$ is called its hypercyclic vector. Here $\mathbb{N}_{0}=\mathbb{N} \cup\{0\}$.

The dynamics of linear operators and, as a special case, the theory of hypercyclic operators were actively developed for the last 20 years. A detailed review of the results up to the end of 1990-s is given in paper [1]. For a recent exposition of the theory, see monographs [2, 3].

However, first examples of hypercyclic operators appeared much earlier. In 1929, Birkhoff has shown that the translation operator $T_{a}: f(z) \mapsto f(z+a), a \in \mathbb{C}, a \neq 0$, is hypercyclic in the Frechet space of all entire functions $\operatorname{Hol}(\mathbb{C})$ with topology of uniform convergence on the compact sets. Later, McLane proved hypercyclicity of the differentiation operator $D: f \mapsto f^{\prime}$ on $\operatorname{Hol}(\mathbb{C})$. The first example of a hypercyclic operator in the Banach setting was given in 1969 by Rolewicz [4 who showed that for each $\lambda \in \mathbb{C},|\lambda|>1$, the operator $\lambda S^{*}$ is hypercyclic on $\ell^{p}\left(\mathbb{N}_{0}\right), 1 \leqslant p<\infty$, where $S^{*}$ is the backward shift on $\ell^{p}\left(\mathbb{N}_{0}\right)$ transforming a vector $x=$ $\left(x_{0}, x_{1}, \ldots, x_{n}, \ldots\right) \in \ell^{p}\left(\mathbb{N}_{0}\right)$ to the vector $\left(x_{1}, x_{2}, \ldots, x_{n+1}, \ldots\right)$.

Given a hypercyclic operator $T$, what can be said about the set of its hypercyclic vectors? Clearly, if $x$ is a hypercyclic vector for operator $T$, then $T x, T^{2} x, T^{3} x, \ldots$ are hypercyclic vectors for $T$ as well. Hence, the set of hypercyclic vectors is dense when it is non-empty.

The following result was proved by Bourdon [5] (a special class of operators commuting with generalized backward shifts was previously considered by Godefroy and Shapiro in [6]).

Theorem (Bourdon, [5]). Let $T$ be a hypercyclic operator acting on a Hilbert space $H$. Then there exists a dense linear subspace, where any non-zero vector is hypercyclic for $T$.

Definition. Given a hypercyclic operator $T$, an infinite-dimensional closed subspace, in which every non-zero vector is hypercyclic for $T$, is called a hypercyclic subspace.

Montes-Rodriguez [7, Theorem 3.4] proved that the operator $\lambda S^{*},|\lambda|>1$, on $\ell^{2}\left(\mathbb{N}_{0}\right)$ has no hypercyclic subspaces. However, for some class of functions of the backward shift $S^{*}$ on $\ell^{2}(\mathbb{N})$ there exists a hypercyclic subspace, and it is the main result of the present paper. To state

A.A. Lishanskit, Existence of hypercyclic subspaces for Toeplitz operators.

(c) Lishanskit A.A. 2015.

THE WORK WAS SUPPORTED BY THE GRANT MD-5758.2015.1 AND BY JSC "GAZPROM NEFT".

Submitted April 20, 2015. 
it, we need to introduce some notations. Let $\mathbb{D}=\{z \in \mathbb{C}:|z|<1\}$ be the unit disc and let $\mathrm{T}=\{z \in \mathbb{C}:|z|=1\}$ be the unit circle. Recall that the disc algebra $A(\mathbb{D})$ is the space of all functions continuous in the closed disc $\overline{\mathbb{D}}$ and analytic in $\mathbb{D}$ (with the norm $\max _{z \in \overline{\mathbb{D}}}|\varphi(z)|$ ).

Main Theorem. For each function $\varphi \in A(\mathbb{D})$ such that $\varphi(\mathbb{T}) \cap \mathbb{T} \neq \emptyset$ and $\varphi(\mathbb{D}) \cap \mathbb{T} \neq \emptyset$ the operator $\varphi\left(S^{*}\right)$ on $\ell^{2}\left(\mathbb{N}_{0}\right)$ has a hypercyclic subspace.

Note that the $\varphi(z)=\lambda z,|\lambda|>1$, does not satisfy this condition.

The examples of applying the Main Theorem may be interpreted as certain Toeplitz operator on the Hardy space. The Hardy space $H^{2}=H^{2}(\mathrm{D})$ is the space of all functions of the form $f(z)=\sum_{n \geqslant 0} c_{n} z^{n}$ with $\left\{c_{n}\right\} \in \ell^{2}\left(\mathbb{N}_{0}\right)$, and thus is naturally identified with $\ell^{2}\left(\mathbb{N}_{0}\right)$. Recall that for a function $\varphi \in L^{\infty}(\mathbb{T})$, the Toeplitz operator $T_{\varphi}$ with the symbol $\varphi$ is defined as $T_{\varphi} f=P_{+}(\varphi f)$, where $P_{+}$stands for the orthogonal projection from $L^{2}(\mathbb{T})$ onto $H^{2}$. Then the backward shift on $S^{*}$ may be identified with the Toeplitz operator $T_{\bar{z}}$. It was shown in [6] that each coanalytic Toeplitz operator $T_{\bar{\varphi}}$ (i.e., $\varphi$ is a bounded analytic function in $\mathbb{D}$ ) is hypercyclic whenever $\varphi(\mathbb{D})$ intersects $\mathbb{T}$. Our Main Theorem provides a class of coanalytic Toeplitz operators having a hypercyclic subspace.

A general sufficient condition for the existence of a hypercyclic subspace was given by Gonzalez, Leon-Saavedra and Montes-Rodriguez in [8]. To state it, we need the following stronger version of hypercyclicity:

Definition. An operator $T$ acting on a separable Banach space $\mathcal{B}$ is said to be hereditarily hypercyclic if there exists a sequence of non-negative integers $\left\{n_{k}\right\}$ such that for each subsequence $\left\{n_{k_{i}}\right\}$ there exists a vector $x$ such that the sequence $\left\{T^{n_{k_{i}} x}\right\}$ is dense in $\mathcal{B}$.

We also need to recall the notion of the essential spectrum.

Definition. An operator $U$ is called Fredholm if $\operatorname{Ran} U$ is closed and has a finite codimension and Ker $U$ is finite-dimensional. The essential spectrum of an operator $T$ is defined as

$$
\sigma_{e}(T)=\{\lambda: T-\lambda I \text { is non-Fredholm }\} .
$$

Theorem (Gonzalez, Leon-Saavedra, Montes-Rodriguez, [8, Theorem 3.2]). Let T be a hereditary hypercyclic bounded linear operator on a separable Banach space $\mathcal{B}$. Let the essential spectrum of $T$ intersect the closed unit disc. Then there exists a hypercyclic subspace for operator $T$.

We intend to use this result in the proof of the Main Theorem.

Let us mention some other results on this topic. In [9], Shkarin proved that the differentiation operator on the standard Frechet space $\operatorname{Hol}(\mathbb{C})$ has a hypercyclic subspace. In [10, Corollary 5.5], Quentin Menet generalized this result: he proved that for each non-constant polynomial $P$ the operator $P(D)$ has a hypercyclic subspace. He also obtained some results concerning weighted shifts on $\ell^{p}$.

\section{ON ESSENTIAL SPECTRA OF LINEAR OPERATORS}

The following lemma is well known. We give its proof for the convenience of the reader.

Lemma. Essential spectrum of the operator $S^{*}$ is the unit circle.

Proof. Let us consider three cases:

Case 1: $|\lambda|>1$. The operator $S^{*}-\lambda I=-\lambda\left(I-\frac{1}{\lambda} S^{*}\right)$ is invertible and, thus, it is Fredholm.

Case 2: $|\lambda|<1$. We have $S^{*}-\lambda I=S^{*}(I-\lambda S)$. Since the operator $S^{*}$ is Fredholm (its kernel is one-dimensional, its image is the whole space $\ell^{2}$ ), and $I-\lambda S$ is invertible, their composition is also a Fredholm operator.

Case 3: $|\lambda|=1$. The operator $S^{*}-\lambda I$ is not Fredholm because its image has an infinite codimension. 
Indeed, the pre-image of the sequence $\left(\lambda y_{1}, \lambda^{2} y_{2}, \lambda^{3} y_{3}, \lambda^{4} y_{4}, \ldots\right) \in \ell^{2}$ is given by $\left(a, \lambda\left(y_{1}+\right.\right.$ a), $\left.\lambda^{2}\left(y_{1}+y_{2}+a\right), \ldots\right)$ and the identity $a=-\sum_{i=1}^{+\infty} y_{i}$ is necessary for the inclusion of this sequence into $\ell^{2}$.

Then the pre-image of the sequence

$$
(1, \frac{1}{2}, \underbrace{0, \ldots, 0}_{\geqslant 2^{2}-1 \text { times }}, \frac{1}{4}, \underbrace{0, \ldots, 0}_{\geqslant 2^{4}-1 \text { times }}, \ldots, \frac{1}{2^{n}}, \underbrace{0, \ldots, 0}_{\geqslant 2^{2^{n}-1 \text { times }}}, \ldots),
$$

multiplied componentwise by $\left(\lambda, \lambda^{2}, \lambda^{3}, \ldots\right)$, is given by

$$
(-2,-1, \underbrace{\frac{1}{2}, \ldots,-\frac{1}{2}}_{\geqslant 2^{2} \text { times }}, \underbrace{-\frac{1}{4}, \ldots,-\frac{1}{4}}_{\geqslant 2^{4} \text { times }}, \ldots, \underbrace{-\frac{1}{2^{n}}, \ldots,-\frac{1}{2^{n}}}_{\geqslant 2^{2^{n}} \text { times }}, \ldots),
$$

multiplied componentwise by $\left(1, \lambda, \lambda^{2}, \ldots\right)$, but such sequences do not belong to $\ell^{2}$. All sequences of the form (1), as is easily seen, form an infinite-dimensional subspace in $\ell^{2}$.

The following important theorem about the mapping of the essential spectra can be found, e.g., in [11, p. 107].

Essential Spectrum Mapping Theorem. For each bounded linear operator $T$ in a Hilbert space $H$ and for each polynomial $P$, one has $\sigma_{e}(P(T))=P\left(\sigma_{e}(T)\right)$.

\section{Proof of the Main Theorem}

In the proof of hereditary hypercyclicity of operator $\varphi\left(S^{*}\right)$ we will use the following wellknown criterion due to Godefroy and Shapiro [6] (for the explicit statement see, e.g., [3, Theorem $3.1])$ :

Theorem (Godefroy-Shapiro criterion). Let $T$ be a bounded linear operator in a separable Banach space. Suppose that the subspaces

$$
\begin{aligned}
X_{0} & =\operatorname{span}\{x \in X: T x=\lambda x \text { for some } \lambda \in \mathbb{C},|\lambda|<1\}, \\
Y_{0} & =\operatorname{span}\{x \in X: T x=\lambda x \text { for some } \lambda \in \mathbb{C},|\lambda|>1\},
\end{aligned}
$$

are dense in $X$. Then $T$ is hereditarily hypercyclic.

Proof of the Main Theorem. We should verify two conditions of the theorem of Gonzalez, LeonSaavedra and Montes-Rodriguez.

Each function $\varphi$ in the disc-algebra can be approximated uniformly in $\overline{\mathrm{D}}$ by a sequence of polynomials $P_{n}$. Thus, $P_{n}\left(S^{*}\right)$ tends to $\varphi\left(S^{*}\right)$ in the operator norm.

We need to show that $\sigma_{e}\left(\varphi\left(S^{*}\right)\right)$ intersects the closed unit disc. Since $\varphi(\mathbb{T}) \cap \mathbb{T} \neq \emptyset$, there exist $\lambda, \mu \in \mathbb{T}$ such that $\varphi(\lambda)=\mu$. Then $\mu_{n}=P_{n}(\lambda)$ tends to $\mu$. By the Essential Spectrum Mapping Theorem, for each polynomial $P$ one has $\sigma_{e}\left(P\left(S^{*}\right)\right)=P\left(\sigma_{e}\left(S^{*}\right)\right)=P(\mathrm{~T})$. In particular, $\mu_{n}=P_{n}(\lambda) \in \sigma_{e}\left(P_{n}\left(S^{*}\right)\right)$ for each $n$, and therefore, $P_{n}\left(S^{*}\right)-\mu_{n} I$ is not Fredholm.

Since the set of Fredholm operators is open in the operator norm (see, e.g., 12, Theorem 4.3.11]), the set of non-Fredholm operators is closed. Hence, the limit of $P_{n}\left(S^{*}\right)-\mu_{n} I$, which is equal to $\varphi\left(S^{*}\right)-\mu I$, is not Fredholm, and $\mu$ belongs to the essential spectrum of $\varphi\left(S^{*}\right)$. The first condition of the theorem by Gonzalez, Leon-Saavedra and Montes-Rodriguez is verified.

It is well known that the condition $\varphi(\mathbb{D}) \cap \mathbb{T} \neq \emptyset$ implies that $\varphi\left(S^{*}\right)$ satisfies the GodefroyShapiro criterion. Let us briefly recall this argument.

Recall that the point spectrum of $S^{*}$ equals $\sigma_{p}\left(S^{*}\right)=\{\lambda:|\lambda|<1\}$ and an eigenvector is given by $\left(1, \lambda, \lambda^{2}, \cdots\right) \in \ell^{2}\left(\mathbb{N}_{0}\right)$, or, if we pass to the Hardy space $H^{2}(\mathbb{D})$ using the natural 
identification of $H^{2}$ with $\ell^{2}\left(\mathbb{N}_{0}\right)$, by

$$
k_{\lambda}(z)=\frac{1}{1-\bar{\lambda} z}=\sum_{n \geqslant 0} \lambda^{n} z^{n} .
$$

These are the Cauchy kernels being reproducing kernels of $H^{2}$. Clearly, $k_{\lambda}, \lambda \in \mathbb{D}$, are also eigenvectors of $\varphi\left(S^{*}\right)$ associated with the eigenvalues $\varphi(\lambda)$.

By the condition $\varphi(\mathbb{D}) \cap \mathbb{T} \neq \emptyset$, we know that $\varphi(\mathbb{D})$ is an open set intersecting both $\mathbb{D}$ and $\mathbb{C} \backslash \bar{D}$. Clearly, both of the sets $X_{0}=\left\{k_{\lambda}, \lambda \in \mathbb{D}:|\varphi(\lambda)|>1\right\}$ and $Y_{0}=\left\{k_{\lambda}, \lambda \in \mathbb{D}:|\varphi(\lambda)|<1\right\}$ are dense in $H^{2}$. Indeed, $f \in H^{2}$ is orthogonal to $k_{\lambda}$ if and only if $f(\lambda)=0$ and both $\{\lambda \in \mathbb{D}:|\varphi(\lambda)|>1\}$ and $\{\lambda \in \mathbb{D}:|\varphi(\lambda)|<1\}$ are open sets. Thus the conditions of the Godefroy-Shapiro criterion are satisfied and the hereditarily hypercyclicity of operator $\varphi\left(S^{*}\right)$ follows.

Thus, by the theorem of Gonzalez, Leon-Saavedra and Montes-Rodriguez, the operator $\varphi\left(S^{*}\right)$ has a hypercyclic subspace.

In conclusion, we formulate one open question. It would be interesting to generalize the statement of Montes-Rodriguez that the operator $\lambda S^{*},|\lambda|>1$, on $\ell^{2}\left(\mathbb{N}_{0}\right)$, has no hypercyclic subspaces. A natural conjecture is:

Conjecture. Let $B=p\left(S^{*}\right)$, where $p$ is a polynomial such that $|p(\lambda)|>1$ for $|\lambda|=1$. Then operator $B$ has no hypercyclic subspaces.

\section{ACKNOWLEDGEMENTS}

The author is grateful to Quentin Menet for helpful comments.

\section{BIBLIOGRAPHY}

1. K.-G. Grosse-Erdmann. Universal families and hypercyclic operators // Bull. Amer. Math. Soc. 36:3, 345-381 (1999).

2. F. Bayart, E. Matheron. Dynamics of linear operators. Cambridge Univ., Cambridge (2009).

3. K.-G. Grosse-Erdmann, A. Peris Manguillot. Linear chaos. Springer, Berlin (2011).

4. S. Rolewicz. On orbits of elements // Studia Math. 32:1, 17-22 (1969).

5. P.S. Bourdon. Invariant manifolds of hypercyclic vectors // Proc. Amer. Math. Soc. 118:3, 845-847 (1993).

6. G. Godefroy, J.H. Shapiro. Operators with dense, invariant, cyclic vector manifolds // J. Funct. Anal. 98:2, 229-269 (1991).

7. A. Montes-Rodriguez. Banach spaces of hypercyclic vectors // Michigan Math. J. 43:3, 419-436 (1996).

8. M. Gonzalez, F. Leon-Saavedra, A. Montes-Rodriguez. Semi-Fredholm Theory: Hypercyclic and supercyclic subspaces // Proc. London Math. Soc. 81:3, 169-189 (2000).

9. S. Shkarin. On the set of hypercyclic vectors for the differentiation operator // Israel J. Math. 180:1, 271-283 (2010).

10. Q. Menet. Hypercyclic subspaces and weighted shifts //Adv. Math. 255, 305-337 (2014).

11. S. Goldberg. Unbounded linear operators. McGraw-Hill, New York (1966).

12. E.B. Davies. Linear operators and their spectra. Cambridge Univ. Press, Cambridge (2007).

Andrei Alexandrovich Lishanskii,

SPbSU, Chebyshev laboratory,

14th Line 29B, Vasilyevsky Island,

St.Petersburg 199178, RUSSIA

E-mail: Lishanskiyaa@gmail.com 